\title{
APLICAÇÃO DA LÓGICA FUZZY PARA CORREÇÃO DE DADOS DE PERFIL DE RAIOS GAMA E CORRELAÇÃO COM POROSIDADE EFETIVA DE TESTEMUNHOS DA BACIA DE RESENDE, RIO DE JANEIRO
}

\author{
Francisco de Assis Leal de Souza ${ }^{1}$, Leonardo Fernandes Tunala', Paula Lúcia Ferrucio da Rocha², \\ Carlos Jorge de Abreu', Ricardo Rhomberg Martins ${ }^{3}$, Inayá Correa Barbosa Lima ${ }^{4}$ \\ e Ricardo Tadeu Lopes ${ }^{1}$ \\ Recebido em 14 dezembro, 2009 / Aceito em 15 abril, 2010 \\ Received on December 14, 2009 / Accepted on April 15, 2010
}

\begin{abstract}
This work presents here a study on the porosity of cores from Resende Basin (Rio de Janeiro). The first step involved the selection, thinning of the material (core) and sample preparation for the use of porosimeter to obtain effective porosity. The material studied is sandstone, friable, coarse to very fine of three wells (GPR1, GPR2 and GPR3). The fuzzy logic (MatLab) was applied to the effective porosity data calculated from the gamma ray (GR) profile for the construction of a synthetic profile and from this we could identify the errors made in calculating the data. Then the results of porosity from the core were correlated with data from the corrected effective porosity (gamma ray profile) in order to prove the results obtained with both methods. The analysis of the synthetic porosity calculated by the fuzzy logic has shown that this is a promising method for assessing the quality of data and to obtain good fits.
\end{abstract}

Keywords: porosity, gamma ray profile, fuzzy logic.

RESUMO. Este trabalho apresenta os resultados de um estudo sobre a porosidade de testemunhos da Formação Resende na região da Bacia de Resende (Rio de Janeiro). A primeira etapa envolveu a seleção, desbaste do material (testemunhos) e preparação da amostra para caracterização no porosímetro e obtenção da porosidade efetiva. 0 material estudado é um arenito, friável, de granulometria grossa a muito fina retirado de três poços (GPR1, GPR2 e GPR3). Com a lógica fuzzy, aplicada aos dados de porosidade efetiva do perfil de raios gama - RG, foi construído um perfil sintético que identificou e corrigiu erros no cálculo da porosidade. Em seguida os resultados de porosidade dos testemunhos do poço GPR1 foram correlacionados aos dados de porosidade efetiva corrigidos a partir do perfil de RG, visando comparar os dois métodos. A análise do perfil sintético de porosidade calculado pela lógica fuzzy mostrou que este é um método promissor para filtrar e avaliar a qualidade de dados melhorando a interpretação de dados de perfilagem.

Palavras-chave: porosidade, perfil de raios gama, lógica fuzzy.

\footnotetext{
${ }^{1}$ Universidade Federal do Rio de Janeiro, Instituto de Geociências / CCMN, Departamento de Geologia / Bloco G, Avenida Athos da Silveira Ramos, 274, Cidade Universitária, Ilha do Fundão, 21941-916 Rio de Janeiro, RJ, Brasil - E-mails: pachoteki@yahoo.com.br; leotunala@hotmail.com; abreu@geologia.ufrj.br; ricardo@lin.ufrj.br

2Universidade Federal do Rio de Janeiro, Avenida Athos da Silveira Ramos, 274, Cidade Universitária, Ilha do Fundão, 21941-916 Rio de Janeiro, RJ, Brasil. Tel./Fax: (21) 2590-8091 / 2598-9481 ramal 4 - E-mail: ferrucio@acd.ufrj.br

3 Universidade Federal do Rio de Janeiro, Escola Politécnica de Engenharia da UFRJ, Avenida Athos da Silveira Ramos, 274, Cidade Universitária, Ilha do Fundão, 21941-916 Rio de Janeiro, RJ, Brasil - E-mail: ricard0301@gmail.com

${ }^{4}$ Universidade Federal do Rio de Janeiro, Centro de Tecnologia, Bloco G, sala 101, Ilha do Fundão, Caixa Postal: 68501, 21945-970 Rio de Janeiro, RJ, Brasil -E-mail: inaya@lin.ufrj.br
} 


\section{INTRODUÇÃo}

Perfis em poços são importantes, pois proporcionam padrões de correlação entre poços vizinhos, confecção de mapas geológicos e definição da geometria dos corpos e ambientes de sedimentação. São utilizados também para definir a espessura das camadas, caracterizar a litologia, o tipo de fluido das camadas, fraturas (zonas de perda de circulação), permeabilidade de camadas, o volume de hidrocarboneto móvel e residual, qualidade da cimentação do revestimento, seleção de zonas para canhoneio e produção de hidrocarbonetos, cálculo da pressão de poros, determinação da inclinação e direção de poços (Nery, 2004).

Com dados de perfilagem pode-se obter a porosidade das formações geológicas. Esta importante propriedade petrofísica refere-se à existência de espaços vazios entre as partículas que compõem as rochas (poros, espaços intragranulares e microporos). A porosidade varia com o tamanho e a forma dos grãos e também com a distribuição espacial dos mesmos. A porosidade pode ser primária (deposicional) ou secundária (pós-deposicional). Uma rocha possui porosidade primária se esta foi originada durante sua deposição ou bioconstrução e porosidade secundária quando foi originada de processos geológicos (desenvolvimento de fraturas, cavidades devido à dissolução em calcários, etc.). A porosidade total, expressa em porcentagem, pode ser calculada pela razão entre os espaços vazios ou 0 volume de vazios $\left(V_{v}\right)$ e 0 volume total $\left(V_{t}\right)$ de uma amostra:

$$
\phi=\frac{V_{v}}{V_{t}} \times 100
$$

Utilizou-se neste trabalho testemunhos da região da Bacia de Resende (RJ) com diâmetros originais entre 4 e $7 \mathrm{~cm}$. A escolha das amostras analisadas seguiu critérios geológicos e foi embasada no trabalho de Almada (2007) que fez correlação dos dados do perfil de $\mathrm{RG}$ e os dados da descrição litológica dos três poços (GPR1, GPR2, GPR3) que distam $6 \mathrm{~m}$ entre si, em uma distribuição triangular equilátera. Essa autora definiu cinco fácies sedimentares, dentre as quais quatro fácies arenosas: Acg, Amg, Amf e Amfs; e uma fácies síltica: S; com a descrição litológica e a interpretação do ambiente de sedimentação sintetizada na Tabela 1.

A perfilagem realizada na região da Bacia de Resende forneceu dados de RG (valor de API e porosidade efetiva) e porosidade sônica. Dos testemunhos disponíveis foi utilizado o material do poço GPR1 cujo valor médio de RG para as fácies arenosas foi de $120^{\circ} \mathrm{API}$. Vale ressaltar que nos dados de porosidade efetiva foram encontrados erros de leituras da ferramenta GR que, provavelmente, são decorrentes de leituras feitas nos intervalos do poço GPR1 intercalados com grande quantidade de folhelho ocasionando valores muito altos de $R G_{\max }$. Este erro é observado em quatorze medidas.

\section{METODOLOGIA}

Os dados de porosidade calculados por medidas de laboratório são baseados na lei de Boyle-Mariotte, que relaciona a variação de volume e pressão de um gás ideal em temperatura constante. Foi utilizado um porosímetro (UltraPoroPerm $500{ }^{\circledR}$ ) que possui uma câmara de gás de volume constante $V_{1}$ (câmara de volume de referência), onde 0 gás nitrogênio é injetado e armazenado a uma pressão $P_{1}$. Esta câmara de gás está ligada à câmara de compressão da amostra de volume $V$. Quando a câmara de compressão da amostra contém um volume de amostra $V_{A}$, ela não pode conter mais do que $V-V_{A}$ de volume de gás. Então, no momento em que a válvula de ligação da câmara de gás com a câmara de compressão é aberta, e o gás nitrogênio é liberado isotermicamente para penetrar na amostra, ocorrendo uma variação de volume $(\Delta V)$ e uma nova pressão $P_{2}$ é medida.

$$
P_{1} V_{1}=P_{2}\left(V_{1}+\Delta V\right)
$$

onde $\Delta V=V-V_{A}$, ou seja, $\Delta V$ é 0 volume de gás na amostra, ou em outras palavras, o volume de espaços porosos na amostra em $\mathrm{cm}^{3}$ (Cupertino, 2005). A Figura 1 apresenta 0 arranjo esquemático de funcionamento.

0 preparo das amostras para 0 ensaio no porosímetro envolveu lapidação manual das amostras, pois, a câmara de confinamento do porosímetro possui diâmetro interno de $2,54 \mathrm{~cm}$ $\left(1^{\prime \prime}\right)$ e $10,16 \mathrm{~cm}\left(4^{\prime \prime}\right)$ de comprimento; os valores de porosidade foram obtidos através da relação entre 0 volume de vazios e 0 volume da amostra. 0 porosímetro tem por função coletar informação numérica sobre os espaços inter e/ou intragranulares $\left(\mathrm{cm}^{3}\right)$ de cada amostra introduzida na câmara de confinamento do equipamento. 0 porosímetro faz o registro dos valores de volume de poros das amostras por variação de pressão na câmara de referência. A Figura 2 apresenta uma foto do equipamento no laboratório.

Para 0 ajuste dos dados do perfil de RG foi utilizada a regra fuzzy (análise do grau de pertinência de dados) que parte do princípio de que se um grupo de dados $x_{k}$ tem vários padrões dentro de si, sendo que cada grupo de padrão individual pode ser agrupado em torno de um centro. Desta forma, espera-se que a distância dos pontos naquele grupo possa ser correlacionada através de uma sequência fuzzy a qual descreve uma matriz de partição $U$ ou membership grade, satisfazendo as seguintes 
Tabela 1 - Fácies sedimentares (Almada, 2007).

\begin{tabular}{|c|l|l|}
\hline Fácies & \multicolumn{1}{|c|}{ Descrição } & \multicolumn{1}{c|}{ Interpretação } \\
\hline Acg & Arenito conglomerático fino maciço & Fluxos em lençol, trativo \\
\hline Amg & Arenito muito grosso a médio & Deposição rápida de carga arenosa \\
\hline Amf & Arenito médio a muito fino maciço & Fluxo de detritos arenosos subaquosos \\
\hline Amfs & Arenito fino a muito fino, mal selecionado & Fluxo gravitacional (corridas de lama) \\
\hline S & Siltito arenoso, bioturbado & Depósito de planície de inundação \\
\hline
\end{tabular}

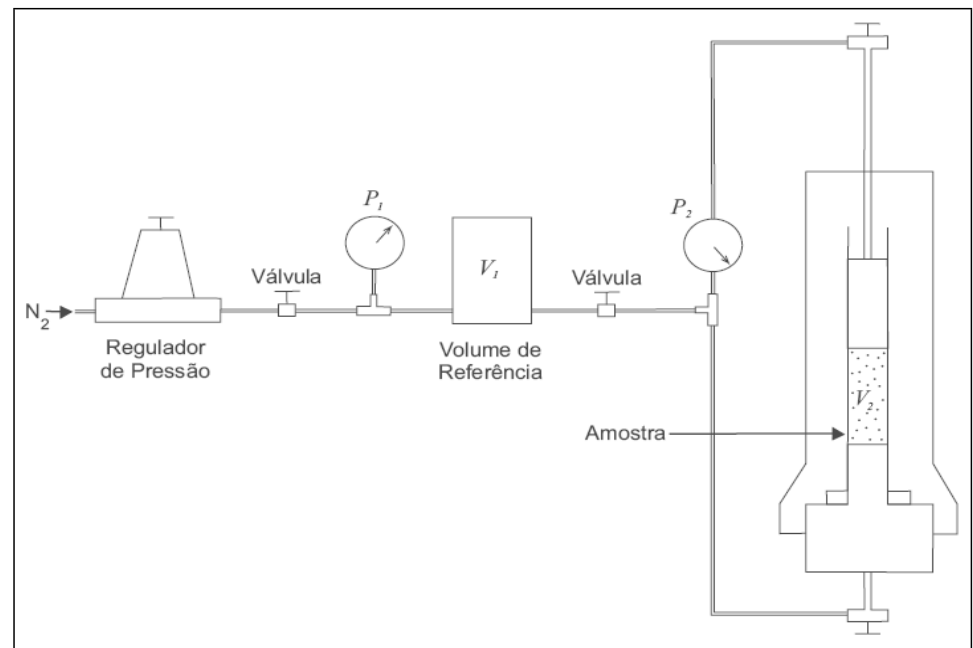

Figura 1 - Esquema de funcionamento do porosímetro (Cupertino, 2005).

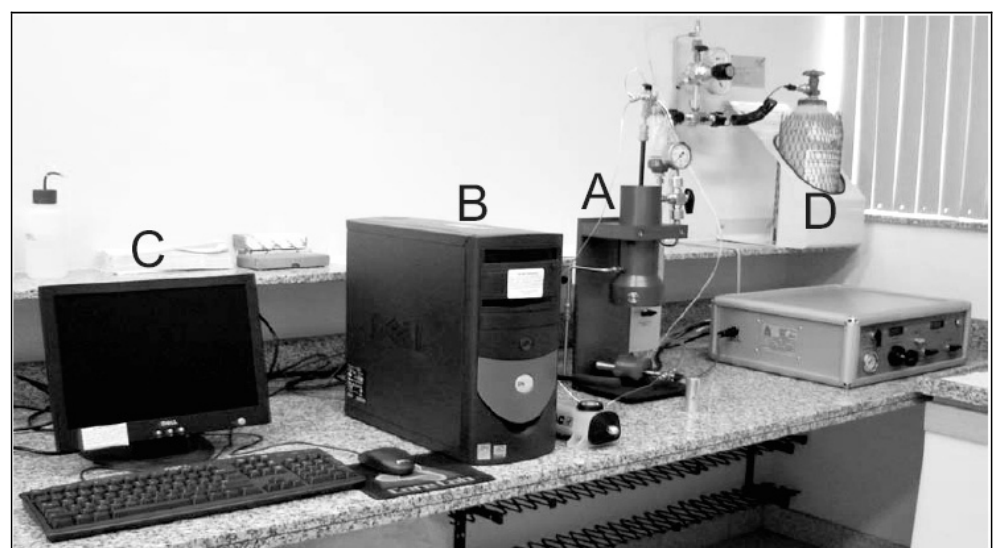

Figura 2 - Fotografia do porosímetro: A) Câmara de confinamento; B) Computador; C) Tela de leitura de dados; D) Cilindro de gás.

condições:

$$
\begin{gathered}
\mu_{i k} \in[0,1] \text { onde } 1 \leq i \leq 3 \text { e } 1 \leq k \leq n \\
\qquad \sum_{i=1}^{c} \mu_{i k}=1 ; \\
0 \leq \sum_{k=1}^{c} \mu_{i k} \leq n,
\end{gathered}
$$

onde $c$ é o número de grupos, e $n$ é o número de dados.

A condição dada pela Equação (3) significa que os dados pertencem a vários grupos e a diferentes graus. Já as condições dadas pelas Equações (4) e (5) requerem apenas que o grau total da matriz de partição para cada dado seja normalizado a 1, e não podem pertencer a mais fácies do que o número existente.

A localização de um grupo (cluster) no gráfico é representada pelo seu valor central $v_{i j}$ em torno do qual seus $p$ elemen- 


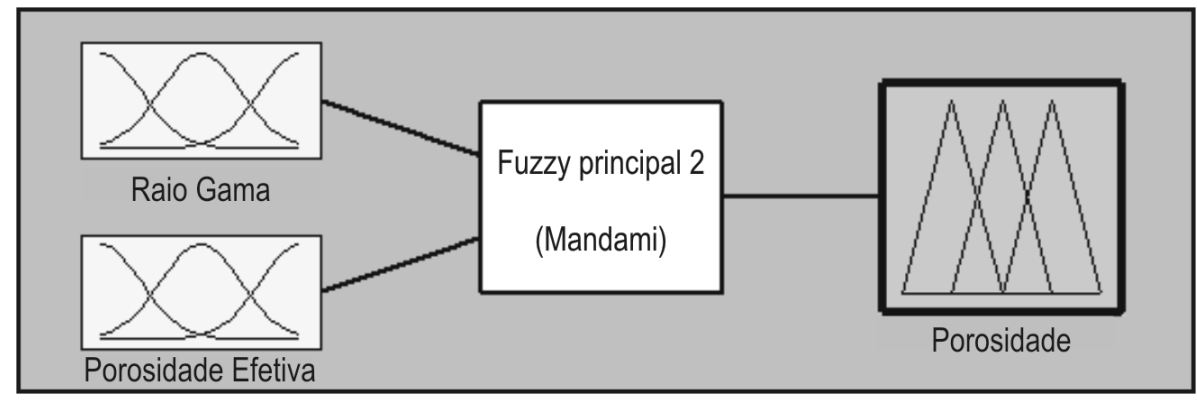

Figura 3 - Fuzificação e Defuzificação: Entradas (RG e Porosidade Efetiva), Sistema (Mandami) e Saída.

tos estão concentrados. 0 critério usado para melhorar a partição inicial é o critério da variância. Neste caso, mede-se a dissimilaridade entre os pontos em um grupo e seu valor central pela distância euclidiana, $d_{i k}$, que é dada por:

$$
d_{i k}=\left\|x_{k}-v_{i}\right\|=\left[\sum_{j=1}^{p}\left(x_{k j}-v_{i j}\right)^{2}\right]^{1 / 2} .
$$

A partição fuzzy, de acordo com este critério, é feita minimizando 0 seguinte funcional ou função objetiva:

$$
F(\mu, v)=\sum_{i=1}^{c} \sum_{k=1}^{n}\left(\mu_{i k}\right)^{m}\left(d_{i k}\right)^{2},
$$

0 que resulta em

$$
\mu_{i k}=\left[\frac{\frac{1}{\left\|x_{k}-v_{i}\right\|^{2}}}{\sum_{j=1}^{c}\left(\frac{1}{\left\|x_{k}-v_{j}\right\|^{2}}\right)}\right]^{\frac{1}{m-1}}
$$

tal que

$$
v_{i}=\frac{1}{\sum_{k=1}^{2}\left(\mu_{i k}\right)^{m}} \sum_{k=1}^{n}\left(\mu_{i k}\right)^{m} x_{k}, \text { para } 1 \leq i \leq c .
$$

0 sistema descrito pelas Equações 6, 7, 8 e 9 não pode ser resolvido analiticamente. A solução recai, então, na aplicação de técnicas de algoritmos iterativos que aproximam o mínimo do funcional a partir de uma dada posição inicial. Um dos algoritmos mais conhecidos para solucionar tal problema é 0 algoritmo ISODATA, proposto por Bezdek (1980) onde, para cada $m \in(0, \infty)$, resolve-se iterativamente as condições necessárias. Pode-se citar também os trabalhos de Hathaway \& Bezdek (2001) e Das Gupta (2001) em aplicações de regra fuzzy.

Neste trabalho utilizou-se o método fuzzy c-means (fcm) no software MatLab ${ }^{\circledR} 7$, para obtenção dos grupos e seus centros a partir dos dados, substituindo 0 algoritmo ISODATA. A função fcm é utilizada para tal, proporciona a possibilidade de análise simplificada para a identificação e escolha dos intervalos dos grupos: [center, $\left.U, f_{-} o b j\right]=f c m($ Data, ncluster $)$. Onde Data é o conjunto de dados e ncluster o número de grupos escolhidos, a função retorna a matriz de valores de grau de pertinência e a matriz de centros dos grupos.

Nesta etapa a primeira tarefa a ser realizada são os agrupamentos fuzzy. Estes são mostrados em gráficos que representam cada grupo definindo seus respectivos centros. Um elemento pode pertencer ou não pertencer parcialmente a um conjunto e 0 que determina o grau de pertinência é a premissa de quanto mais próximo estiver do centro deste conjunto mais o elemento vai pertencer ao conjunto.

As entradas iniciais para o sistema fuzzy são os valores de API do perfil de RG, a porosidade efetiva e porosidade sônica, tais dados de porosidade foram obtidos na perfilagem feita na região de estudo (empresa Perfil Master). Após a determinação dos intervalos e do número de inputs (entradas) do sistema procede-se à construção do sistema fuzzy no aplicativo MatLab ${ }^{\circledR} 7$ conforme exemplifica a Figura 3.

\section{RESULTADOS}

Os resultados de porosidade obtidos com o porosímetro estão na Tabela 2. A porosidade dos testemunhos está entre $18,161 \%$ e $37,972 \%$ no GPR1, $10,236 \%$ a $37,580 \%$ no GPR2 e $16,291 \%$ a $35,406 \%$ para 0 GPR3. Com médias individuais de 26,839\%, 23,435\% e 23,641\% respectivamente para GPR1, GPR2 e GPR3. A porosidade média das 48 amostras é $24,638 \%$.

Os resultados do agrupamento para o perfil sintético utilizando lógica fuzzy são apresentados na Figura 4 ( $A, B$ e C). Os grupos de cores distintas com seus respectivos centros e os limites dos intervalos projetados sobre os eixos determinam a base de dados para as regras de inferência no sistema fuzzy. As regras para inferência no sistema fuzzy (unidade de decisão lógica) são determinadas a partir da relação entre os 
Tabela 2 - Porosidade dos testemunhos dos poços GPR1, GPR2 e GPR3 obtidos no porosímetro.

\begin{tabular}{|c|c|c|c|c|}
\hline Profundidade & Amostras & Porosidade GPR1 & Porosidade GPR2 & Porosidade GPR3 \\
\hline $2,00(\mathrm{~m})$ & AM01 & $27,983 \%$ & $28,158 \%$ & $25,720 \%$ \\
\hline $6,00(\mathrm{~m})$ & AM02 & $19,251 \%$ & $19,259 \%$ & $16,291 \%$ \\
\hline $9,00(\mathrm{~m})$ & AM03 & $35,710 \%$ & $29,044 \%$ & $31,186 \%$ \\
\hline $12,00(\mathrm{~m})$ & AM04 & $30,363 \%$ & $30,606 \%$ & $27,736 \%$ \\
\hline $14,65(\mathrm{~m})$ & AM05 & $23,728 \%$ & $12,590 \%$ & $18,121 \%$ \\
\hline $17,00(\mathrm{~m})$ & AM06 & $23,456 \%$ & $21,812 \%$ & $16,604 \%$ \\
\hline $20,00(\mathrm{~m})$ & AM07 & $28,510 \%$ & $31,174 \%$ & $20,063 \%$ \\
\hline $24,00(\mathrm{~m})$ & AM08 & $18,398 \%$ & $15,870 \%$ & $17,787 \%$ \\
\hline $27,00(\mathrm{~m})$ & AM09 & $27,225 \%$ & $32,908 \%$ & $31,630 \%$ \\
\hline $29,00(\mathrm{~m})$ & AM10 & $25,884 \%$ & $26,023 \%$ & $21,349 \%$ \\
\hline $33,00(\mathrm{~m})$ & AM11 & $18,161 \%$ & $14,782 \%$ & $16,764 \%$ \\
\hline $36,00(\mathrm{~m})$ & AM12 & $37,972 \%$ & $37,580 \%$ & $28,002 \%$ \\
\hline $37,00(\mathrm{~m})$ & AM13 & $31,693 \%$ & $34,820 \%$ & $28,391 \%$ \\
\hline $41,00(\mathrm{~m})$ & AM14 & $27,084 \%$ & $10,510 \%$ & $17,006 \%$ \\
\hline $45,00(\mathrm{~m})$ & AM15 & $27,944 \%$ & $10,236 \%$ & $35,406 \%$ \\
\hline $45,70(\mathrm{~m})$ & AM16 & $26,056 \%$ & $19,577 \%$ & $26,190 \%$ \\
\hline
\end{tabular}
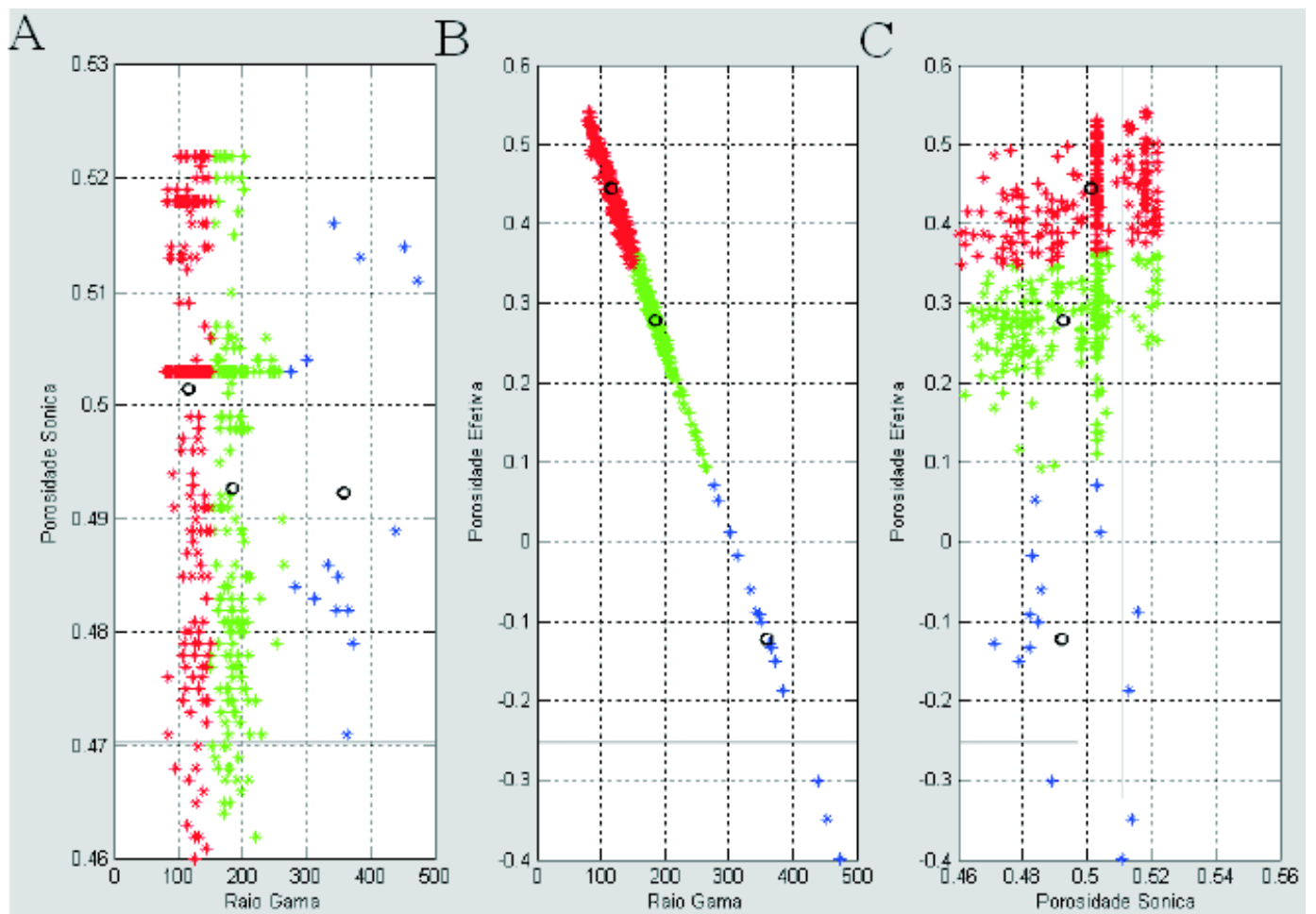

Figura 4 - Agrupamentos dos dados de entrada. Gráfico A: RG $\times$ Porosidade Sônica; Gráfico B: RG $\times$ Porosidade Efetiva; e Gráfico C: Porosidade Sônica $\times$ Porosidade Efetiva. 
grupos (variáveis linguísticas) e seus intervalos projetados nos eixos $x$ e $y$. Assim, para 0 perfil de raios gama, as variáveis linguísticas estabelecidas são: alto, médio e baixo (Tab. 3), pois está associado à alta concentração de elementos radioativos na Formação Resende.

Tabela 3 - Variável linguística e dados de entrada fuzzy.

\begin{tabular}{|c|c|c|c|}
\hline $\begin{array}{c}\text { Variável } \\
\text { Linguística }\end{array}$ & $\mathrm{RG}$ & $\begin{array}{c}\text { Porosidade } \\
\text { Efetiva }\end{array}$ & $\begin{array}{c}\text { Porosidade } \\
\text { Sônica }\end{array}$ \\
\hline Muito Alto & & $\mathrm{x}$ & $\mathrm{x}$ \\
\hline Alto & $\mathrm{x}$ & $\mathrm{x}$ & $\mathrm{x}$ \\
\hline Médio & $\mathrm{x}$ & $\mathrm{x}$ & $\mathrm{x}$ \\
\hline Baixo & $\mathrm{x}$ & $\mathrm{x}$ \\
\hline
\end{tabular}

A porosidade sônica com valores entre 46,2 e 52,2\% foi analisada pelo sistema fuzzy; tais dados não apresentaram possibilidade de utilização num sistema de agrupamento fuzzy (Fig. 4). Entretanto, caso a porosidade sônica fosse analisada, sua definição como variável linguística para o sistema fuzzy na região do poço GPR1, seria: muito alto, alto e médio (Tab. 3). Devido aos dados de "altos" valores de porosidade sônica não foi considerado 0 valor baixo. Por outro lado, os dados de porosidade efetiva apresentaram bom ajustamento com a base de regras fuzzy. Portanto, as variáveis fuzzy estipuladas para porosidade efetiva foram alto, médio e baixo (Tab. 3).

A distribuição dos dados entrada fuzzy está nos gráficos $\mathrm{A}$, B e C da Figura 4. 0 gráfico A (RG $\times$ porosidade sônica) apresenta dois agrupamentos (vermelho e verde) dispostos ao longo do eixo vertical $(0,46$ a 0,53) que não se distinguem, pois há sobreposição de dados de porosidade sônica.

0 gráfico 4B (porosidade efetiva $\times$ raios gama) apresenta um padrão linear diagonal decrescente com relação ao eixo horizontal $\left(0^{\circ}\right.$ a $\left.500^{\circ} \mathrm{API}\right)$ e com os centros (euclidianos) dentro de cada um dos grupos. 0 agrupamento dos dados é crescente com relação ao eixo da porosidade e, demonstra que as regras de inferência podem ser obtidas a partir do comportamento dos grupos neste gráfico.

Podemos observar neste mesmo gráfico (4B) que para a faixa entre $80^{\circ}$ a $150^{\circ} \mathrm{API} 0$ grupo vermelho (porosidade efetiva) está aproximadamente entre 34 e $55 \%$, e a verificação dos dados de porosidade efetiva da perfilagem mostra que mais da metade dos valores de porosidade efetiva está entre $34,1 \%$ e $54,2 \%$ (raios gama entre $79,4^{\circ}$ e $168,3^{\circ}$ ) e decrescem conforme 0 aumento dos valores de RG. Portanto, a porosidade é alta quando os valores de RG são relativamente "baixos". Vale lembrar, que os "altos" valores de grau API e porosidade efetiva aqui encontrados, são valores relativos devido aos prováveis erros da ferramenta no momento da leitura.

0 gráfico $\mathrm{C}$ apresenta o mesmo comportamento do gráfico $\mathrm{A}$, pois, não há visualização definida dos grupos nos eixos horizontal e vertical, como no gráfico B. Não representa um padrão que relacione os dados de porosidade. De acordo com as análises feitas nos gráficos $A, B$ e $C$ da Figura 4 as entradas que podem ser escolhidas para o sistema de inferência fuzzy são os dados de porosidade efetiva $\times R G$, pois estes dados apresentam 0 tipo de distribuição que melhor se ajusta ao sistema de inferência fuzzy.

Além da distribuição dos dados de entrada (Fig. 4), outra informação importante para o sistema de inferência fuzzy é o tipo de distribuição destes. Neste caso, o contorno das barras dos histogramas da Figura 5 é semelhante a triângulos e, portanto, define 0 modelo de distribuição (triangular) dos dados para serem defuzificados pela unidade de decisão lógica.

As regras de inferência para 0 sistema fuzzy estão na Tabela 4 e foram estabelecidas com base na relação entre as variáveis fuzzy descritas na Tabela 3. 0 sistema fuzzy determina através do sistema de inferência fuzzy - FIS os novos valores de porosidade como saída. A Tabela 4 mostra as regras de inferência.

0 sistema de inferência fuzzy escolhido é do tipo Mandami (Fig. 6), com duas entradas (porosidade efetiva e RG) e uma saída. 0 resultado final é uma saída de dados de porosidade gerados a partir destas variáveis fuzzy. Os intervalos (ranges) para as funções de pertinência das entradas foram obtidos a partir dos intervalos entre as classes do gráfico B da Figura 4 que está representado na Figura 7.

Os intervalos de saída para as funções de pertinência foram estipulados aleatoriamente, dividindo a faixa total em três grupos $(-11 \%$ a $45 \%)$ conforme a Figura 8.

0 resultado da defuzificação é um gráfico que compara 0 novo rol de dados de porosidade com a porosidade obtida no porosímetro conforme é visualizado no gráfico $\mathrm{C}$ da Figura 9. Mas antes de qualquer avaliação convém citar os valores que compreendem os percentuais de porosidades efetiva (dados do perfil) e a porosidade efetiva obtida no porosímetro. São elas respectivamente: de $-39,9 \%$ até $54,2 \%$ (média de $35,0009 \%$ ) e de 18,161 até $37,972 \%$.

0 gráfico $\mathrm{C}$ da Figura 9 mostra o resultado da defuzificação do sistema de inferência fuzzy proposto. Este perfil de porosidade fuzzy ou saída fuzzy foi comparado com perfil de porosidade obtido no porosímetro conforme mostra a Figura 10. Devido à alta quantidade de ruído presente nos dados da defu- 


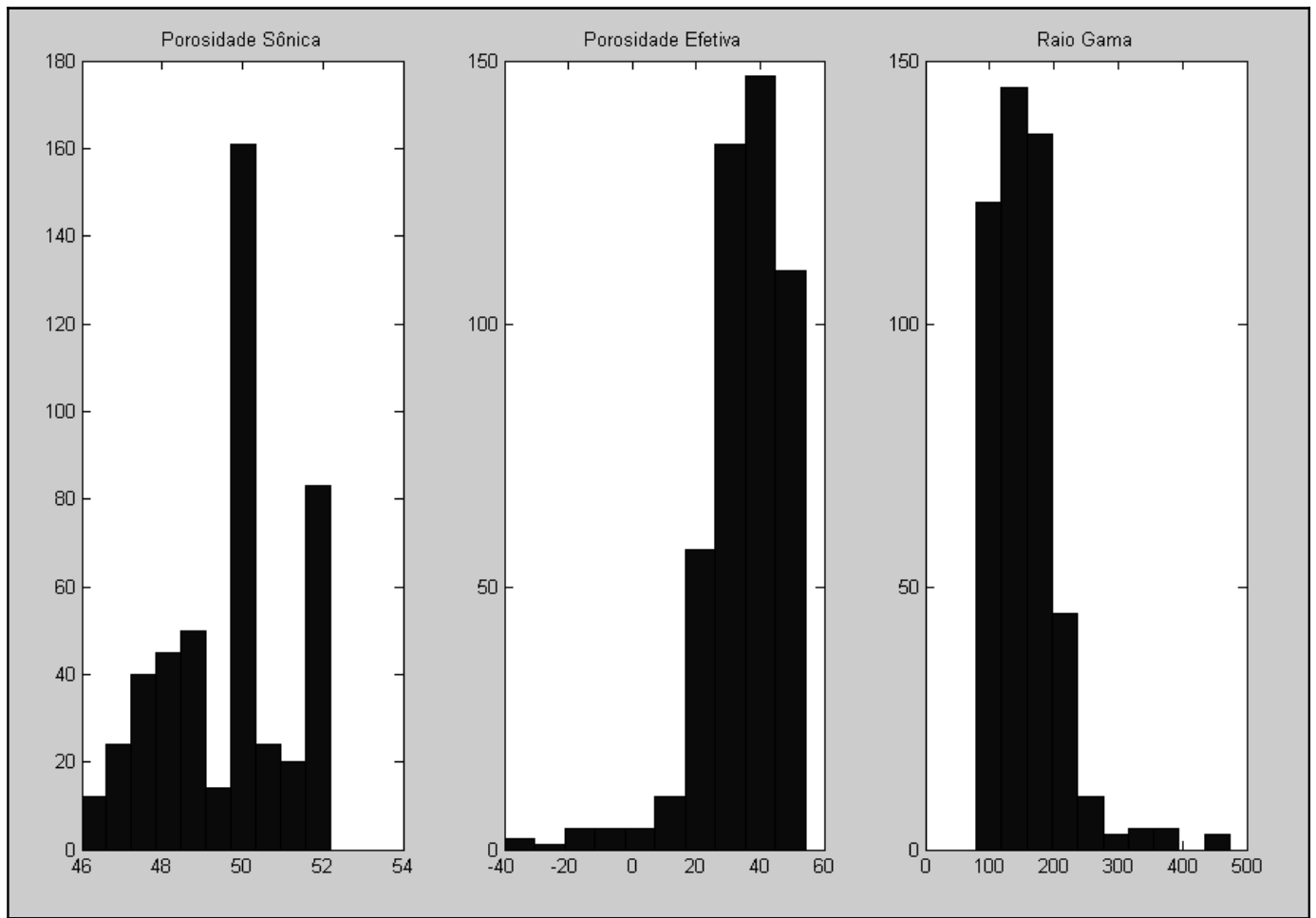

Figura 5 - Histogramas de frequência dos dados de Porosidade Sônica (\%), Porosidade Efetiva (\%) e Raios Gama (GAPI); indicando probabilidade triangular.

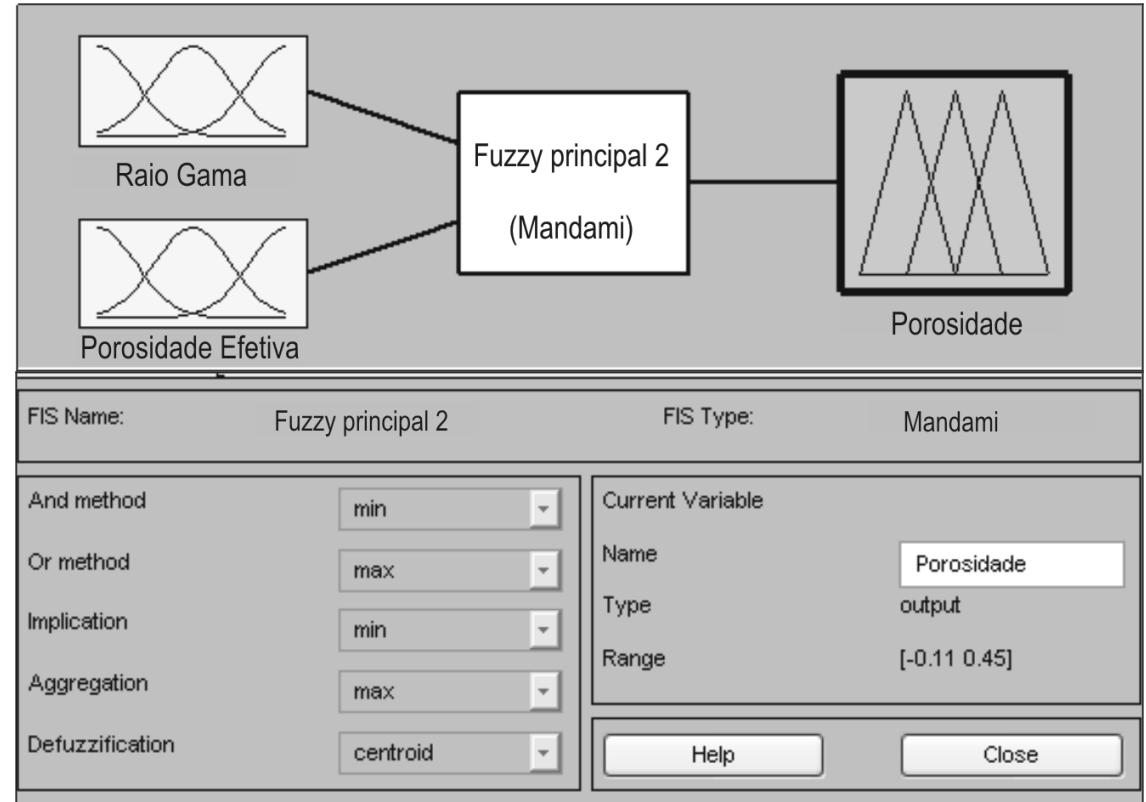

System "Fuzzyprincipal2": 2 inputs, 1 output, and 3 rules

Figura 6 - Modelo Mandami de Defuzificação proposto para os inputs: RG e Porosidade Efetiva do GPR1. 
(a)

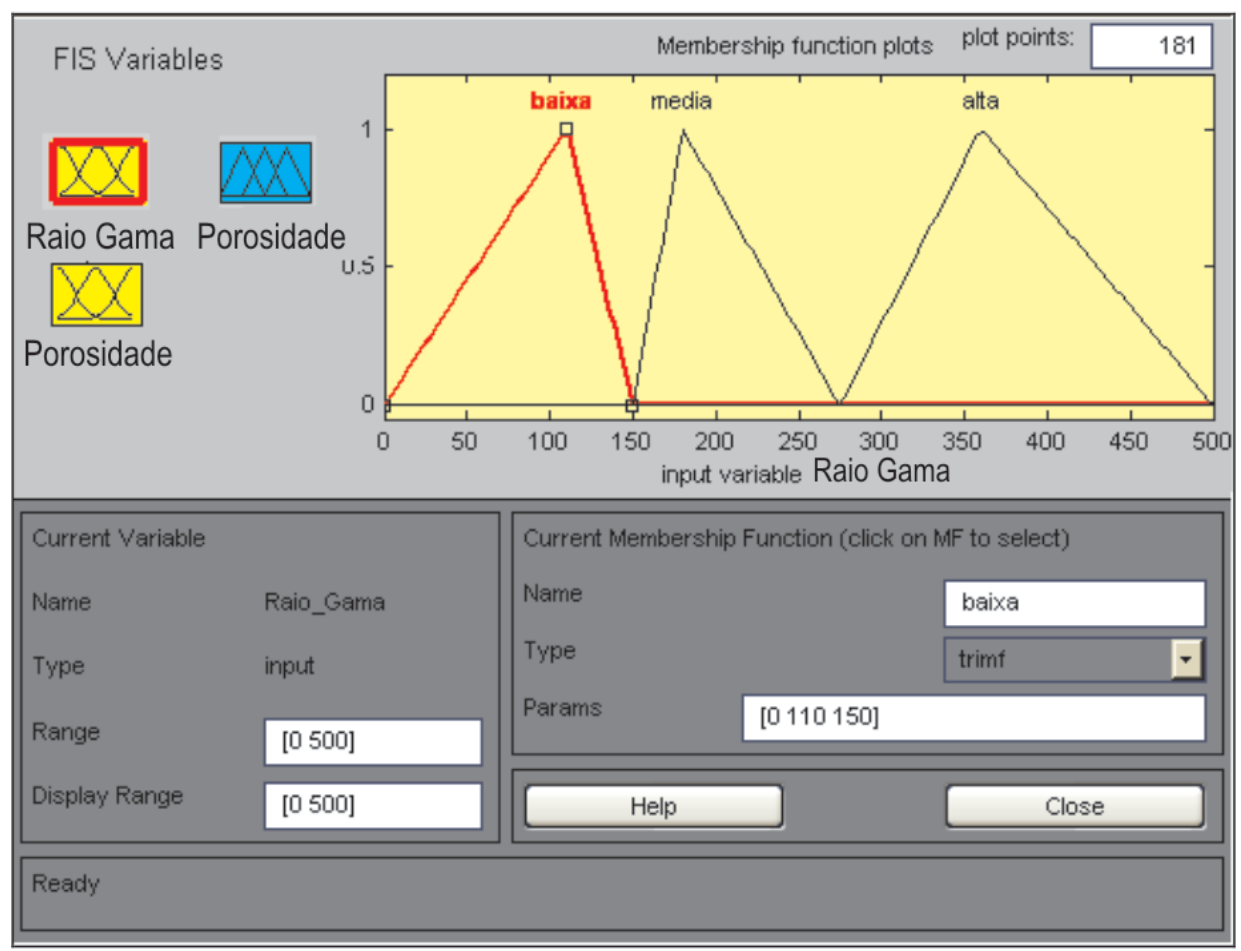

(b)

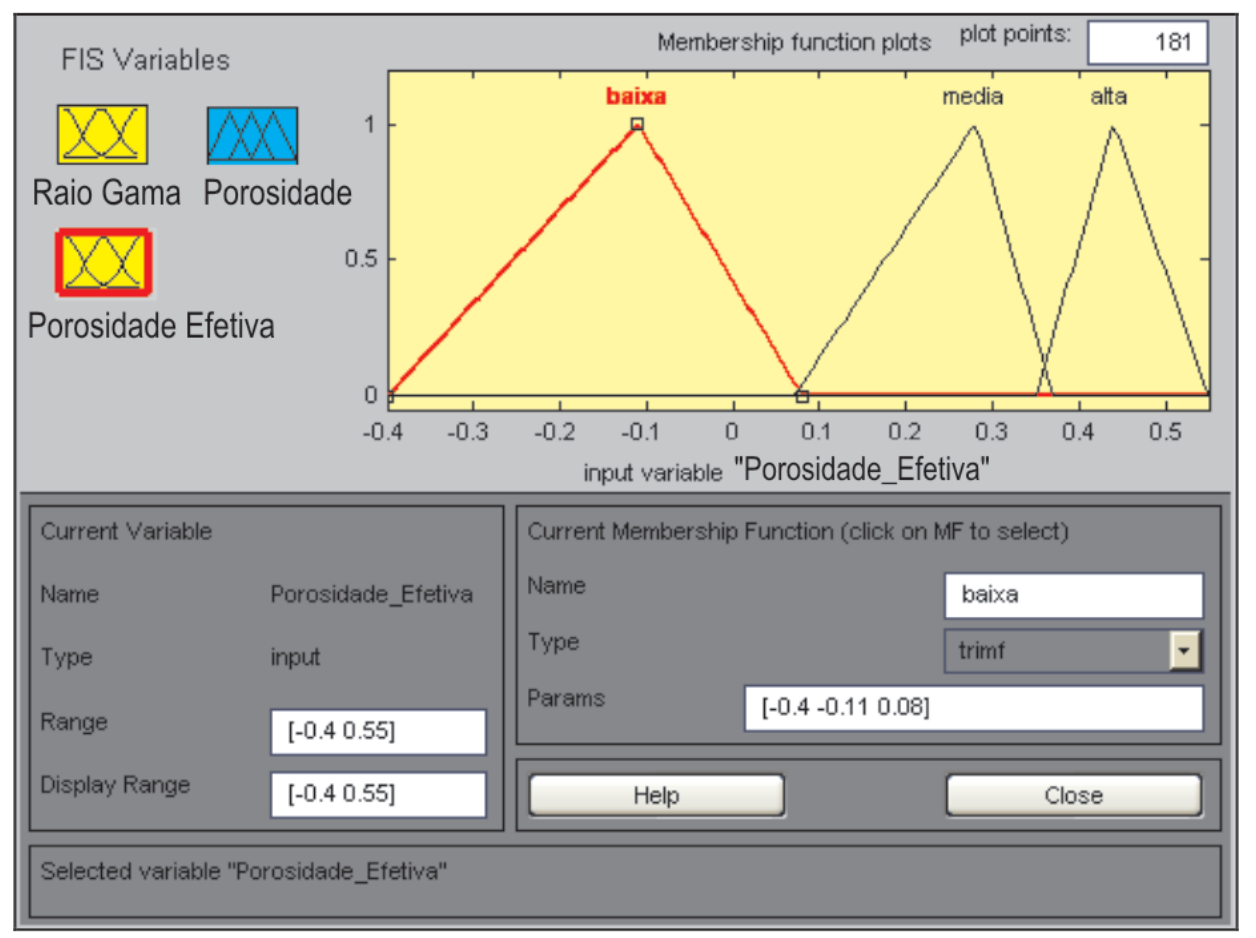

Figura 7 - (a) Intervalo de classe para os dados de entrada de RG; (b) Intervalo de classe para os dados de entrada de Porosidade Efetiva. 
Tabela 4 - Variáveis fuzzy.

\begin{tabular}{|c|c|c|}
\hline Se & e & Então \\
\hline Raio gama é alto & Porosidade Efetiva baixa & Porosidade baixa \\
\hline Raio gama é médio & Porosidade Efetiva média & Porosidade média \\
\hline Raio gama é baixo & Porosidade Efetiva alta & Porosidade alta \\
\hline
\end{tabular}

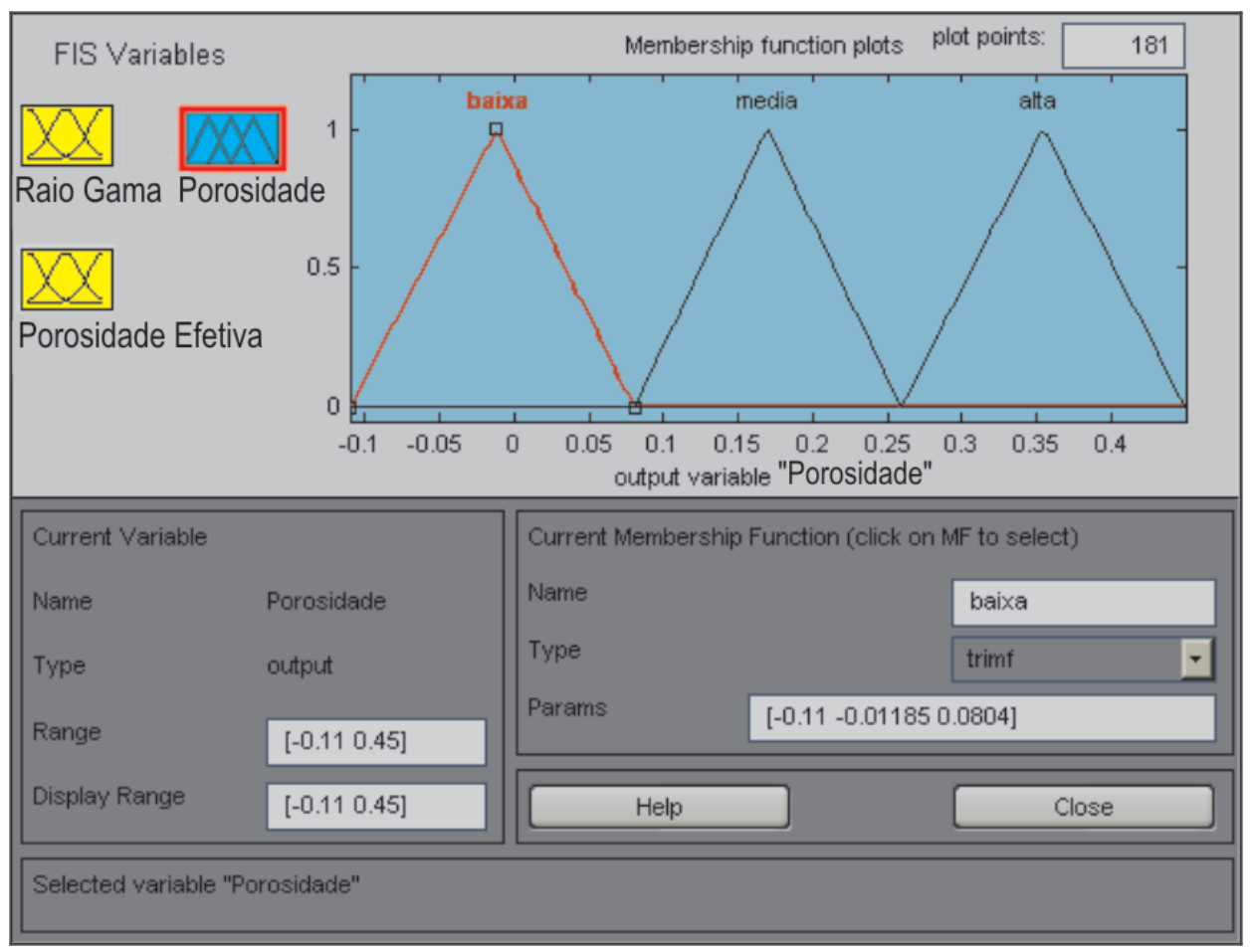

Figura 8 - Intervalos de classe para os dados de saída da Porosidade Efetiva (Defuzificação).

zificação efetuou-se uma suavização com o método de spline cúbico. Este método de ajuste é utilizado quando se torna necessário selecionar dados intermediários desejados e, automaticamente, excluir dados em excedentes. Neste caso, a interpolação foi feita como meio de evitar a interferência de ruídos na defuzificação (Fig. 10).

0 perfil de porosidade fuzzy é convergente com as medidas do porosímetro, e, ao comparar o resultado obtido com os valores de porosidade total do porosímetro (18,161 a 37,972\%) e de porosidade efetiva ( $-39,9$ a $54,2 \%$, entrada fuzzy).

\section{CONCLUSÃO}

Este trabalho apresentou uma simulação de um perfil sintético de porosidade construído através da regra fuzzy com base nos dados de porosidade efetiva do perfil de RG e calibrado com medidas de porosidade efetiva obtidas em laboratório. 0 sistema de inferência fuzzy organizou os dados de porosidade em grupos que apresentaram melhor ajuste quando relacionados ao RG do GPR1 (utilizando para o cálculo da porosidade na unidade lógica do sistema fuzzy os seguintes grupos: porosidade alta, média e baixa). 0 perfil sintético de porosidade não apresenta grandes discrepâncias em suas curvas e oscila entre 17 e $35 \%$ denotando bom ajuste com a porosidade efetiva (obtida em porosímetro), bastando para isso comparar os valores de porosidade entre $18,161 \%$ até $37,972 \%$ com os valores acima descritos da defuzificação. No perfil sintético (regra fuzzy) é possível observar que o método foi capaz de reconhecer os valores negativos incorretos que existiam no dado original de porosidade efetiva e fez a exclusão de tais valores por estarem fora do centro do grupo (cluster) apresentando baixo grau de pertinência fuzzy. A interpretação do resultado fuzzy 

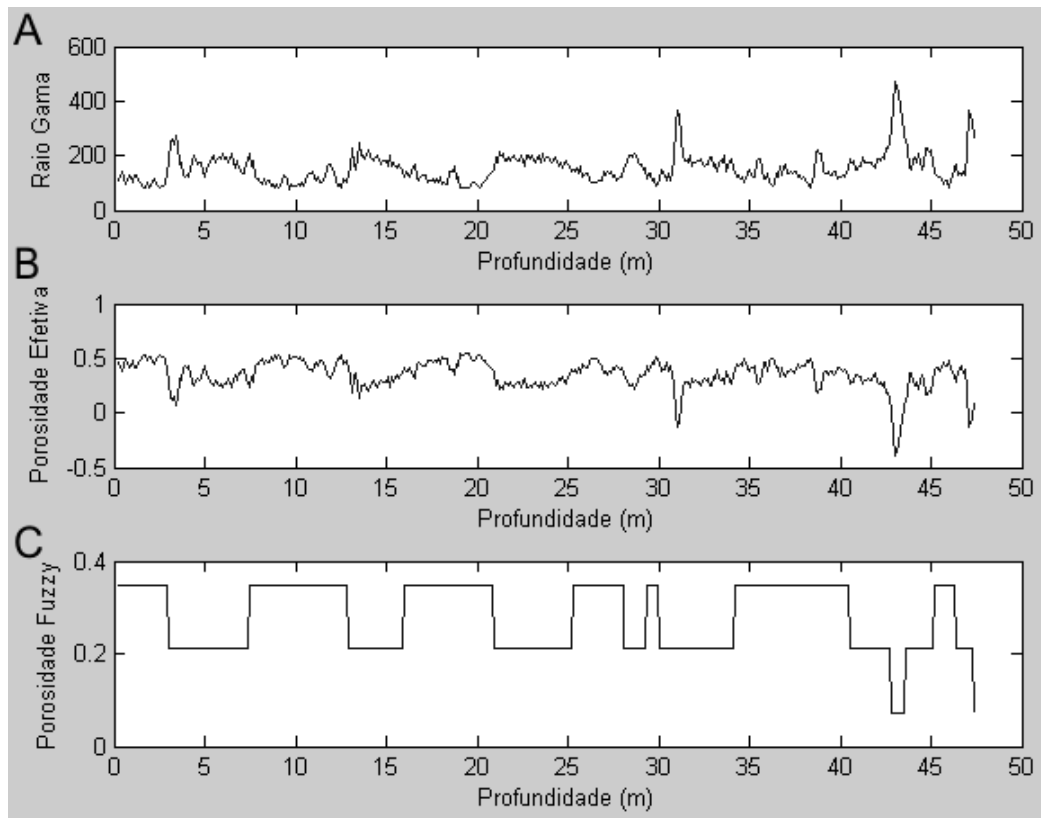

Figura 9 - Resultado da Defuzificação. Gráficos A (RG) e B (Porosidade Efetiva) entradas fuzzy e Gráfico C (saída fuzzy).

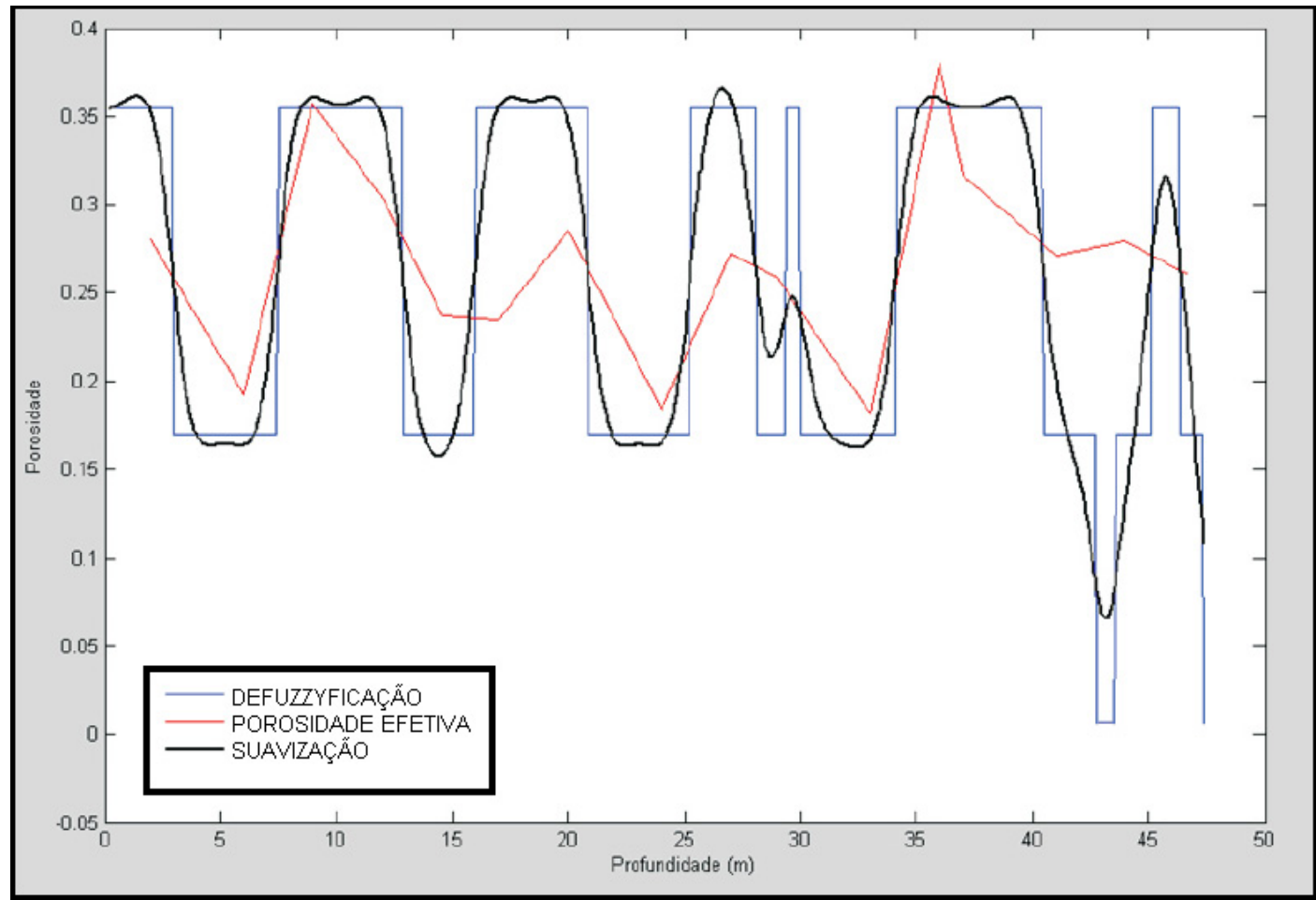

Figura 10 - Defuzificação: dados de Porosidade Efetiva correlacionados com dados de Porosidade Total obtidos com o porosímetro. Legenda: Curva Azul = Defuzificação ou saída fuzzy, Curva Vermelha $=$ Porosidade Total e Curva Preta = Spline cúbico ou suavização.

(defuzificação) está na 'otimização' da porosidade efetiva. Este é um fator muito positivo e promissor para a confecção de perfis sintéticos, mais ainda quando se observa os valores de média do perfil sintético (26\%) que é muito próximo da média de porosidade efetiva do porosímetro (28,06\%).

Portanto, tais observações nos levam a concluir a eficiência 
e a confiabilidade do sistema fuzzy para o tratamento de dados de poço e confecção de perfis sintéticos. Tal correlação entre as curvas (defuzificação e porosidade efetiva) mostra 0 grande ajuste do resultado obtido pela aplicação da lógica fuzzy com os valores medidos usando o porosímetro. Portanto, este é um método promissor de se obter um resultado único a partir de diferentes informações obtidas por diferentes métodos de medição, principalmente pela grande capacidade de tratamento e seleção da regra fuzzy para de previsão de resultados.

\section{AGRADECIMENTOS}

Aos Professores Giovanni Chaves Stael e Jadir da Conceição da Silva (in memoriam) pelas discussões e sugestões. À Petrobras e ANP pela bolsa de estudos do projeto ANPETRO 11.125.

\section{REFERÊNCIAS}

ALMADA A. 2007. Determinação de Fácies Sedimentares com Base em Dados de Subsuperfície na Formação Resende (Bacia de Resende, RJ) -
Rio de Janeiro, Trabalho de conclusão de curso de Geologia, IGeo/UFRJ. Monografia, $45 \mathrm{p}$.

BEZDEK JC. 1980. A convergence theorem for the fuzzy ISODATA clustering algorithm. IEEE Transactions on Pattern Analysis and Machine Intelligence, Vol. PAMI-2, p. 1-8.

CUPERTINO DF. 2005. Caracterização de propriedades do meio poroso em solo arenoso através de permo-porosímetro a gás - Rio de Janeiro. Trabalho de conclusão de curso de Geologia, IGeo/UFRJ. Monografia, $61 \mathrm{p}$.

DAS GUPTA SP. 2001. Application of a fuzzy pattern recognition method in borehole geophysics. Computers \& Geosciences, 27: 85-89.

HATHAWAY RJ \& BEZDEK JC. 2001. Fuzzy c-means clustering of incomplete data. Systems, Man and Cybernetics, Part B: Cybernetics, IEEE Transactions, 31(5): 735-744.

NERY GG. 2004. Apostila sobre Perfilagem Geofísica. Disponível em: <http://www.geraldogirao.com>. Acesso em: 04 mar. 2010.

\section{NOTAS SOBRE OS AUTORES}

Francisco de Assis Leal de Souza. Graduado em Matemática pela Universidade Federal Rural do Rio de Janeiro. Cursando o $9^{\circ}$ período de Geologia na Universidade do Estado do Rio de Janeiro. Mestre em Ciências (Geologia) pela Universidade Federal do Rio de Janeiro. Cursando o doutorado em Ciências (Geologia) na Universidade Federal do Rio de Janeiro. Atividades: Perfil de poços, MatLab e Regra Fuzzy. Áreas de interesse: Perfilagem Geofísica de Poços, Petrofísica (Porosidade), Matemática Aplicada (Regra Fuzzy).

Leonardo Fernandes Tunala. Graduado em Engenharia Química pela Universidade Federal Rural do Rio de Janeiro. Mestre em Engenharia Química pela Universidade Federal Rural do Rio de Janeiro. Área de interesse: Matemática Aplicada (Regra Fuzzy).

Paula Lúcia Ferrucio da Rocha. Graduada em Física (1985) e em Geologia (1990) pela Universidade Federal do Rio de Janeiro. Mestre em Ciências (Geofísica Métodos Potenciais) pela UFRJ, Doutora em Ciências (Geofísica - Métodos Potenciais) pelo ON/CNPq. Atividades de Pesquisa: Professor de Geofísica na Universidade Federal do Rio de Janeiro. Áreas de interesse: Perfilagem Geofísica de Poços, Petrofísica (Ressonância Magnética Nuclear), GPR e Meio Ambiente.

Carlos Jorge de Abreu. Graduado em Geologia pela Universidade de Brasília (UnB). Mestre em Ciências pela The University of Cincinnati, Ohio, USA. Doutor em Ciências pela Universidade Federal do Rio de Janeiro. Atividades de Pesquisa: Professor de Geologia na Universidade Federal do Rio de Janeiro, Instituto de Geociências, Departamento de Geologia. Áreas de interesse: Geologia de Petróleo e Sedimentologia.

Ricardo Rhomberg Martins. Graduação em Engenharia Eletrônica pela Escola de Engenharia da UFRJ, mestrado em Engenharia Elétrica pelo Instituto Alberto Luiz Coimbra de Pós-Graduação e Pesquisa de Engenharia. Doutorado em Engenharia Elétrica pelo Instituto Alberto Luiz Coimbra de Pós-Graduação e Pesquisa de Engenharia. Atualmente é Professor da Escola de Engenharia da UFRJ. Atuando principalmente nos seguintes temas: Sistemas Híbridos, Sistemas Fotovoltaicos, Lógica Fuzzy.

Inayá Correa Barbosa Lima. Graduação em Física pela Universidade Federal Fluminense. Mestrado e doutorado em Engenharia Nuclear pela Universidade Federal do Rio de Janeiro. Tem experiência na área de Física Aplicada, com ênfase em Tomografia Computadorizada 3D e Fluorescência de raios X, atuando principalmente em análises não destrutivas para investigação de parâmetros estruturais internos de estruturas complexas. Atualmente é Professora Adjunta do Departamento de Engenharia Mecânica e Energia - Instituto Politécnico da UERJ e pesquisadora da COPPE-UFRJ.

Ricardo Tadeu Lopes. Graduação em Física pela Universidade Federal do Paraná. Mestrado em Engenharia Nuclear pela Universidade Federal do Rio de Janeiro. Doutorado em Engenharia Nuclear pela Universidade Federal do Rio de Janeiro. Atualmente é Professor Titular da Universidade Federal do Rio de Janeiro. Tem experiência na área de Engenharia Nuclear, com ênfase em Aplicações de Radioisótopos, atuando principalmente nos seguintes temas: Tomografia Computadorizada, Fluorescência de raios X, Difração de raios X e Radiografia Digital. 